\title{
Évaluation des ressources en eau de la Martinique : calcul spatialisé de la pluie efficace et validation à l'échelle du bassin versant
}

\section{Assessment of Martinique (FWI) water resources: effective rainfall spatial modelling and validation at catchment scale}

\author{
Benoit Vittecoq, Patrick Lachassagne, Sandra Lanini et Jean Christophe \\ Maréchal
}

Volume 23, numéro 4, 2010

URI : https://id.erudit.org/iderudit/045098ar

DOI : https://doi.org/10.7202/045098ar

Aller au sommaire du numéro

Éditeur(s)

Université du Québec - INRS-Eau, Terre et Environnement (INRS-ETE)

ISSN

0992-7158 (imprimé)

1718-8598 (numérique)

Découvrir la revue

Citer cet article

Vittecoq, B., Lachassagne, P., Lanini, S. \& Maréchal, J. (2010). Évaluation des ressources en eau de la Martinique : calcul spatialisé de la pluie efficace et validation à l'échelle du bassin versant. Revue des sciences de l'eau / Journal of Water Science, 23(4), 361-373. https://doi.org/10.7202/045098ar
Résumé de l'article

L'évaluation des différents termes du bilan hydrologique à l'échelle d'un bassin versant constitue l'un des points clés de la gestion des ressources en eau, et ce, tout particulièrement dans les régions montagneuses présentant de fortes variations spatiales de la pluviométrie et de l'évapotranspiration. Une méthodologie, basée sur le modèle classique de Thornthwaite, est proposée. Elle prend en compte les différents types de sols, l'occupation des sols ainsi que les effets topographiques et calcule les différents termes du bilan hydrologique (pluie, évapotranspiration, pluie efficace, etc.). L’approche a été mise en oeuvre à l'échelle du kilomètre carré, pour l'ensemble de l'île de la Martinique $\left(1080 \mathrm{~km}^{2}\right)$, puis validée à l'échelle du bassin versant, en comparant les pluies efficaces calculées avec les débits mesurés aux stations de jaugeage. Malgré l'absence de calage des différents paramètres du modèle, les résultats sont très satisfaisants. Une surestimation de la pluie efficace est néanmoins observée pour la plupart des bassins versants utilisés pour la validation du modèle. Cet écart est attribué à une sous-estimation de l'évapotranspiration potentielle, la plupart des bassins versants comportant une composante forestière significative, non prise en compte dans le modèle. 


\title{
ÉVALUATION DES RESSOURCES EN EAU DE LA MARTINIQUE : CALCUL SPATIALISÉ DE LA PLUIE EFFICACE ET VALIDATION À L'ÉCHELLE DU BASSIN VERSANT
}

Assessment of Martinique (FWI) water resources: effective rainfall spatial modelling and validation at catchment scale

\begin{abstract}
BENOIT VITTECOQ ${ }^{1,4 *}$, PATRICK LACHASSAGNE ${ }^{2,3}$, SANDRA LANINI ${ }^{2}$, JEAN CHRISTOPHE MARÉCHAL ${ }^{2}$
\end{abstract}
${ }^{1}$ BRGM, Service Géologique Régional de Martinique, Route Pointe des Nègres, 4 Lot. Miramar, 97200 Fort de France

${ }^{2}$ BRGM, Service EAU/RMD, 1039, rue de Pinville, 34000 Montpellier, France

${ }^{3}$ Actuellement à Evian, Volvic Sources, Danone Eaux France, BP 87, 74500 Evian Cedex, France

${ }^{4}$ Actuellement à BRGM, Service EAU/GRI, 3, avenue Claude Guillemin, BP 6009, 45060 Orléans Cedex 2, France

Reçu le 8 février 2008, accepté le 19 janvier 2010

\section{RÉSUMÉ}

Lévaluation des différents termes du bilan hydrologique à l'échelle d'un bassin versant constitue l'un des points clés de la gestion des ressources en eau, et ce, tout particulièrement dans les régions montagneuses présentant de fortes variations spatiales de la pluviométrie et de l'évapotranspiration. Une méthodologie, basée sur le modèle classique de Thornthwaite, est proposée. Elle prend en compte les différents types de sols, l'occupation des sols ainsi que les effets topographiques et calcule les différents termes du bilan hydrologique (pluie, évapotranspiration, pluie efficace, etc.). L'approche a été mise en œuvre à l'échelle du kilomètre carré, pour l'ensemble de l'île de la Martinique $\left(1080 \mathrm{~km}^{2}\right)$, puis validée à l'échelle du bassin versant, en comparant les pluies efficaces calculées avec les débits mesurés aux stations de jaugeage. Malgré l'absence de calage des différents paramètres du modèle, les résultats sont très satisfaisants. Une surestimation de la pluie efficace est néanmoins observée pour la plupart des bassins versants utilisés pour la validation du modèle. Cet écart est attribué à une sous-estimation de l'évapotranspiration potentielle, la plupart des bassins versants comportant une composante forestière significative, non prise en compte dans le modèle.

\section{Mots clés : pluie efficace, Thornthwaite, modélisation, bassin} versant, bilan hydrologique, ressource en eau, Martinique

\begin{abstract}
The assessment of the various components of the hydrologic budget at catchment scale represents a key challenge for water resources management. This is especially true for regions characterized by important spatial variability in rainfall or evapotranspiration due, for example, to topographical effects. A methodology, based on the classical Thornthwaite model, is proposed to account for soil types, land cover changes and topographical effects on the main components of the water cycle (rainfall, evapotranspiration and efficient rainfall). The
\end{abstract}


approach is developed for the whole Martinique Island (French West Indies, $1080 \mathrm{~km}^{2}$ ) using a $1-\mathrm{km}^{2}$ resolution and validated at catchment scale comparing computed efficient rainfall with measured discharge at several gauging stations. Despite the absence of any calibration of the model parameters, the results are satisfying. A slight overestimation of the efficient rainfall is generally observed for the validation watersheds. This discrepancy is interpreted as an underestimation of potential evapotranspiration as the classical Penman-Montheit formula for grass is used despite the presence of forested areas in most of the watersheds.

\section{Keywords: efficient rainfall, Thornthwaite, modelling, watershed, hydrological budget, water resources, Marti- nique}

\section{INTRODUCTION}

Toute évaluation des ressources en eau à l'échelle d'un territoire nécessite une connaissance la plus précise possible des différents termes du bilan hydrologique. Parmi ceux-ci, la pluie efficace des hydrologues et des hydrogéologues (Peff) correspond, dans son acception la plus large ( $c f$. Glossaire International d'Hydrologie: http://www.cig.ensmp.fr/-hubert/ glu/HINDFRT.HTM), à la " partie de la pluie qui contribue au ruissellement ", soit aux variations de stockage dans le sol près, $\mathrm{P}-\mathrm{ETR}$, où $\mathrm{P}$ correspond aux précipitations et ETR à l'évapotranspiration réelle (en agriculture, sa définition est sensiblement différente puisqu'elle correspond à la " partie de la pluie qui reste dans le sol et contribue au développement des cultures»). Ce terme du bilan est de tout premier intérêt, car il conditionne au premier chef la disponibilité en eau, que ce soit vis-à-vis de son transfert rapide en surface (" ruissellement ») ou de son écoulement différé au travers des aquifères. L'évaluation de ces différents termes du bilan hydrologique, et notamment de la pluie efficace, est tout particulièrement délicate dans les régions montagneuses, qui présentent souvent une forte variabilité spatiale de la pluviométrie ainsi que des variations significatives de la nature des sols, du couvert végétal et de l'évapotranspiration, comme c'est tout particulièrement le cas à la Martinique (Figure 1 et chapitre 2 ci-dessous).

En métropole, des modélisations hydrologiques sont notamment développées par Météo-France. Il s'agit en particulier du schéma de paramétrisation des Interactions Sol - Biosphère - Atmosphère (ISBA) (HABETS et al., 2008; NOILHAN et PLANTON, 1989; NOILHAN et MAHFOUF, 1996), qui constitue la composante "surface " des modèles numériques français de prévision météorologique. ISBA est également utilisé pour des études hydrométéorologiques au sein de la chaine SAFRAN-ISBA-MODCOU qui comprend un système d'analyse météorologique (SAFRAN), le modèle de surface ISBA et un modèle hydrogéologique (HABETS et al., 2008). Cette chaine est utilisée pour disposer d'un monitorage à l'échelle nationale des ressources en eau et pour prévoir les risques d'inondation et de sécheresse à l'échelle du pays. Elle procède au bilan hydrique et au bilan d'énergie au pas de temps horaire et fonctionne avec un maillage de huit kilomètres de côté (soit une superficie de $64 \mathrm{~km}^{2}$ pour chaque maille). Le modèle SAFRAN (forçage atmosphérique) permet notamment l'interpolation et l'extrapolation des données de précipitation observées, en tenant compte notamment des effets orographiques. Ces différents outils ne sont pas encore disponibles dans les départements et régions d'outre-mer. En outre, le maillage actuel de $8 \mathrm{~km}$ de côté s'avèrerait très vraisemblablement insuffisant pour la plupart des applications opérationnelles, compte tenu de la petite taille des bassins versants et de la forte variabilité spatiale des différents termes du bilan et des paramètres nécessaires au calcul.

De ce fait, dans le cadre d'un projet visant à évaluer, pour le Conseil Régional de la Martinique, les ressources en eau souterraine de cette région monodépartementale, une méthode d'évaluation des différents termes du bilan hydrologique, et notamment des pluies efficaces, a été développée par VITTECOQ et al., (2007). Cette méthode s'appuie sur l'établissement de bilans hydrologiques à l'échelle de mailles d'un kilomètre carré de superficie, dont les résultats sont ensuite agrégés en fonction des besoins pour simuler le fonctionnement des bassins versants à étudier. Cette agrégation permet en outre de valider le modèle en comparant les débits simulés aux valeurs observées aux stations de jaugeage.

\section{LA MARTINIQUE}

La Martinique, Département français d'outre-mer, est située sous le tropique du Cancer par 14³0' de latitude nord et $61^{\circ}$ de longitude ouest (Figure 1). Elle occupe une position centrale dans l'archipel des Petites Antilles, entre les îles indépendantes de la Dominique au nord et de Sainte Lucie au sud, dont elle est séparée par deux « canaux » marins étroits $(20-30 \mathrm{~km})$ et profonds (entre 1000 et $2000 \mathrm{~m})$. Elle est baignée par l'océan Atlantique à l'est et par la mer des Caraïbes à l'ouest. Avec ses $1080 \mathrm{~km}^{2}$ de superficie, elle forme la plus grande île de l'archipel : $65 \mathrm{~km}$ d'allongement maximum dans l'axe NNW-SSE; $30 \mathrm{~km}$ dans la plus grande largeur, se réduisant à $12 \mathrm{~km}$ à la faveur des échancrures que constituent les baies de Fort-de-France, du Robert et du François.

La nature essentiellement volcanique de la Martinique lui confère un relief accentué. Elle est montagneuse au nord et à l'ouest, au niveau des grands volcans récents. La Montagne Pelée, stratovolcan actif d'environ $15 \mathrm{~km}$ de diamètre, constitue le point culminant de l'île $(1397 \mathrm{~m})$, à son extrémité nord. 


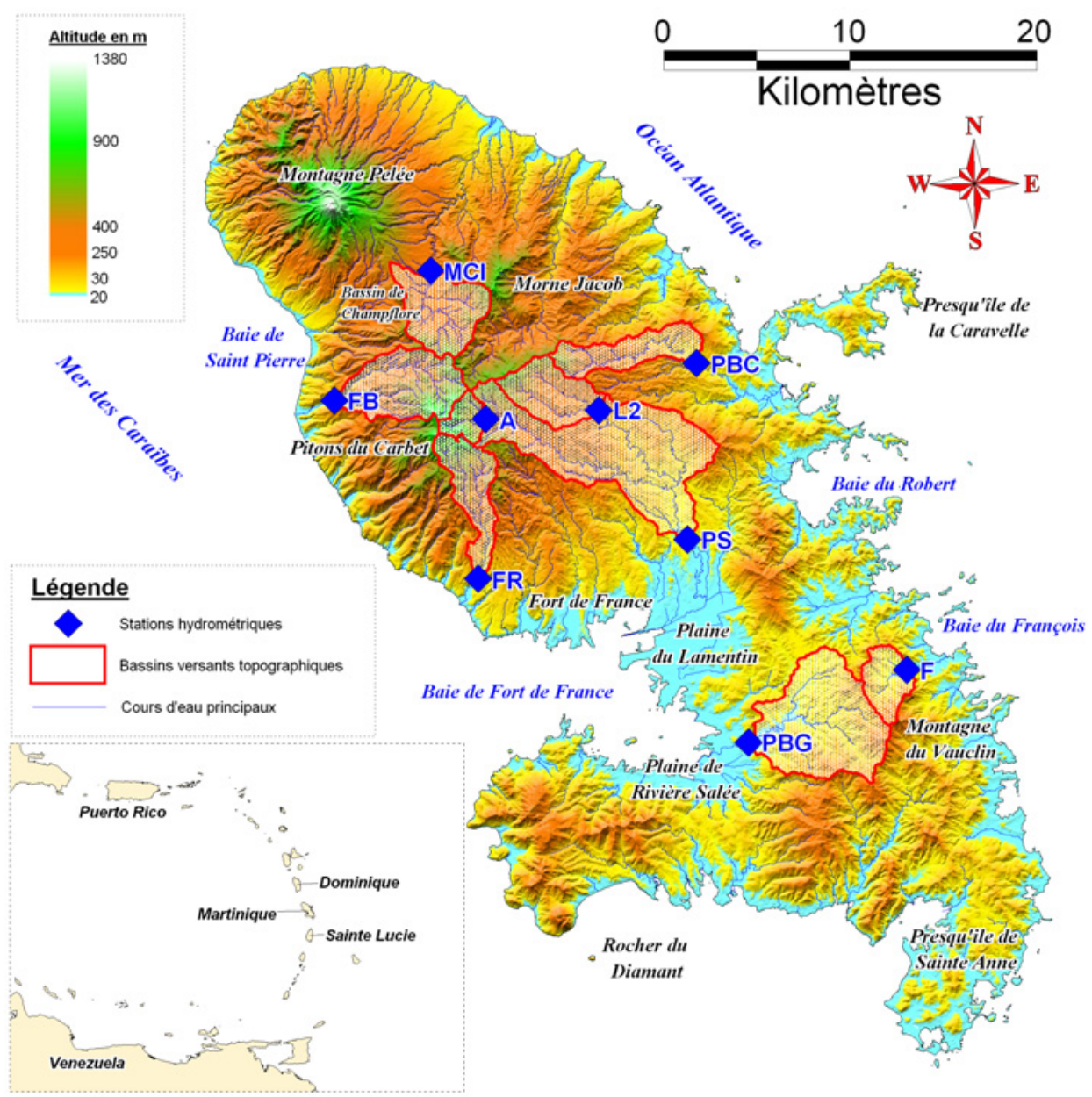

Figure 1. La Martinique : relief (MNT @bdtopo), toponymie simplifiée et localisation des stations hydrométriques et des bassins versants étudiés. Martinique (FWI): topography (Cbdtopo DEM), simplified toponymy and location of the studied gauging stations and the associated surface watersheds.

L'ancien volcan bouclier basaltique et andésitique du Morne Jacob, de plus de $20 \mathrm{~km}$ de diamètre et à la forme surbaissée, occupe toute la partie centrale de l'île. Il culmine encore à $884 \mathrm{~m}$ au droit du sommet éponyme. Sur le flanc ouest de cet édifice, six sommets appartenant au massif des pitons du Carbet, bien que profondément entaillé par l'érosion, dépassent $1000 \mathrm{~m}$ (point culminant Piton Lacroix, 1196 m). Le relief de l'île est plus doux à l'est et au sud, au niveau des complexes volcaniques et volcano-sédimentaires anciens, formés de collines et de petites montagnes ne dépassant pas $500 \mathrm{~m}$ d'altitude (la montagne du Vauclin, avec $504 \mathrm{~m}$, constitue le point culminant du sud de l'île). La "plaine » du Lamentin et de rivière Salée, située dans le prolongement de la baie de Fort-de-France, est occupée par des formations sédimentaires récentes associées au développement d'une végétation de type "mangrove » et constitue la seule zone plane d'extension significative de l'île.

La Martinique bénéficie d'un climat de type tropical humide d'alizés (GUISCAFRE, (1976). La variation thermique saisonnière est relativement faible et inférieure à l'amplitude diurne. Les températures extrêmes annuelles (ALBERT et SPIESER, 1999) varient entre 18 et $32^{\circ} \mathrm{C}$ à Fort-de-France (moyennes de janvier : $21^{\circ} \mathrm{C}-27^{\circ} \mathrm{C}$; moyennes de septembre $24^{\circ} \mathrm{C}-30^{\circ} \mathrm{C}$ ). Le régime des précipitations est caractérisé par une saison sèche ou "carême ", centrée sur les mois de février (moyenne interannuelle $65 \mathrm{~mm}$ à Fort-de-France) à avril, et une saison humide ou " hivernage " centrée sur les mois de juillet à octobre (moyenne interannuelle $255 \mathrm{~mm}$ en septembre à Fort-de-France) et coïncidant avec la période cyclonique. L'intensité des précipitations peut être forte : jusqu'à plusieurs centaines de $\mathrm{mm}$ en moins de $24 \mathrm{~h}$. Le régime des alizés (vents soufflant de l'est) et le relief conditionnent fortement la répartition spatiale de la pluie (Figure 2). Les précipitations moyennes annuelles sont inférieures à $1500 \mathrm{~mm}$ par an dans les zones de faible relief du sud et de l'est de l'île (presqu'îles de Sainte-Anne et de la Caravelle). Elles croissent très fortement avec l'altitude pour atteindre $5000 \mathrm{~mm}$ par an au droit des pitons du Carbet, du bassin de Champflore et, dans une moindre mesure, de la montagne Pelée dont le relief, relativement aigu, constitue un moindre facteur de pluviosité. L'effet de foehn 

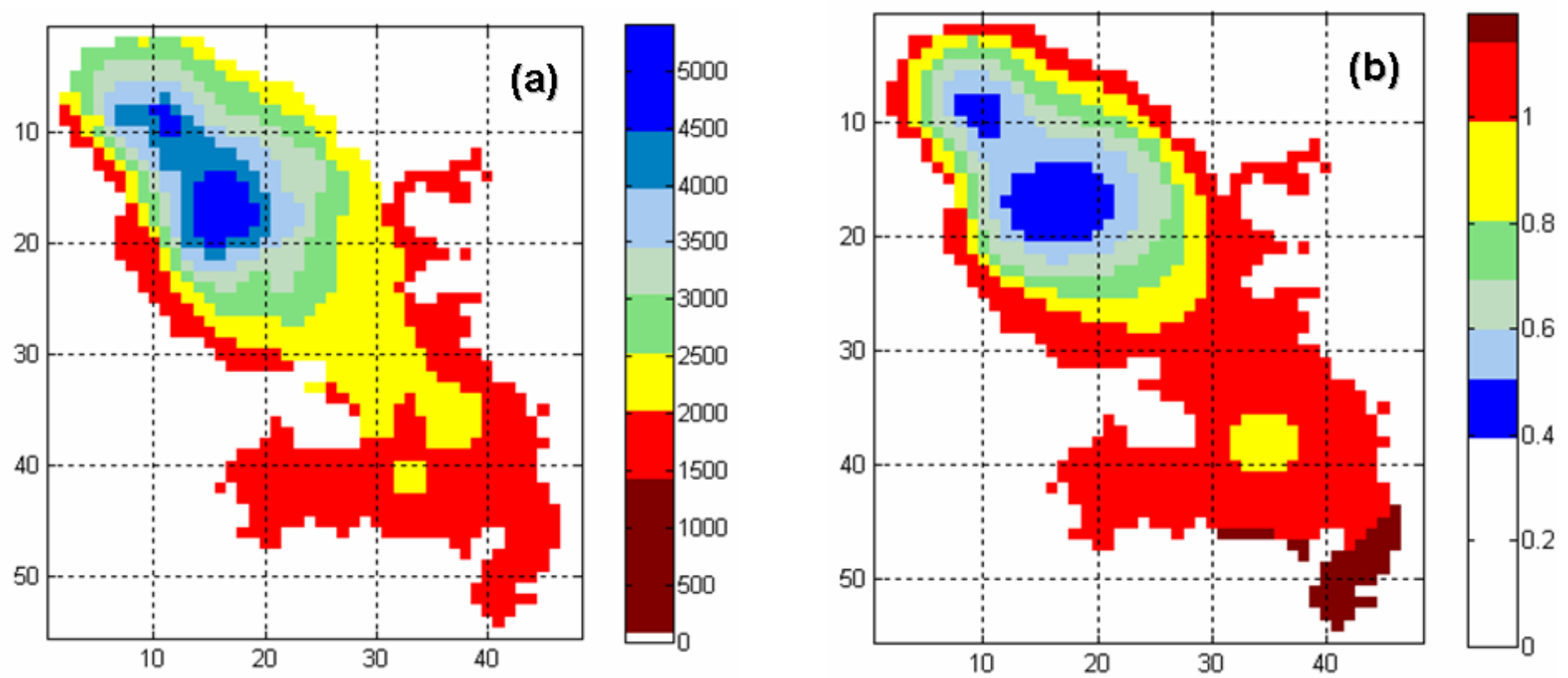

Figure 2. (a) : Isohyètes moyennes annuelles (mm) pour la période 1915-2006; (b) : Iso-ETP moyennes annuelles normées (rapportées à la valeur moyenne annuelle de l'ETP à Fort-de-France $(1670 \mathrm{~mm})$ ). (a): 1915-2006 mean annual rainfall (mm); (b): normalized mean annual ETP (reference: mean annual ETP at Fort-de-France (1670 mm)).

(synonyme alpin du " chinook " nord-américain) associé au relief est à l'origine de la faible pluviosité relevée le long de la côte caraïbe, en particulier sous le vent des sommets du nord de l'île.

La carte pédologique de la Martinique réalisée par l'IRD (http://collaboratif.ird.fr/ezpublish/index.php/IRDMartinique/ content/download/43425/138115/version/3/file/carte_pedo_ SIG972.pdf, COLMET-DAAGE et al., (1969) et COLMETDAAGE (1969)) permet de distinguer les grands types de sols développés en Martinique, qui résultent majoritairement des processus d'altération climatique, avec apparition plus ou moins grande de smectite, en milieu confiné, et de kaolinite, en milieu drainé (WESTERCAMP et al., 1989) : les sols à alluvions, les sols à allophanes relativement peu évolués (sols sur cendres et ponces), les sols peu évolués sur cendres, les ferrisols compacts (palésols fersiallitiques), les sols brun rouille à halloysite, les sols rouges ou bruns montmorillonitiques (sols fersiallitiques) et les sols vertiques et mollisols (vertisols ou sols à argiles gonflantes). Les vertisols (s.l.) sont présents dans les zones sèches situées au vent ou dans les secteurs sous le vent où le déficit hydrique est relativement important. Ils sont caractérisés par un pédoclimat très contrasté, alternant périodes de dessiccation intense (développement de larges fissures) et périodes de sursaturation en eau. Les ferrisols et les sols brun rouille dominent dans les régions humides. Dans la partie nord de l'île, où les cendres et ponces récentes sont très présentes (retombées aériennes notamment), les sols à allophane sont bien développés. Ils sont très poreux et perméables et se caractérisent par des teneurs en eau souvent élevées.

\section{MODALITÉS DE CALCUL DE LA PLUIE EFFICACE}

\subsection{Principe du calcul de la pluie efficace}

La pluie efficace (Peff) et l'évapotranspiration réelle (ETR) ont été calculées par bilan hydrologique au moyen du modèle de Thornthwaite (THORNTHWAITE, 1948) (Figure 3) sur l'ensemble de la Martinique et au pas de temps journalier.

Le principe du modèle de Thornthwaite, brièvement rappelé ci-après, repose sur l'hypothèse que l'ETR est toujours inférieure ou égale à l'EvapoTranspiration Potentielle (ETP). Ainsi, à chaque pas de temps de calcul (journalier dans le cas présent), on vérifie si l'ETP peut être satisfaite ou non, en "puisant " sur les Précipitations (P) ou, si celles-ci sont insuffisantes, sur la Réserve Utile (RU) du sol. Si P > ETP, alors ETR = ETP. Sinon $(\mathrm{P}<\mathrm{ETP})$, l'ETR dépend de la quantité d'eau disponible au sein de la RU; si elle est suffisante, $\mathrm{ETR}=\mathrm{ETP}$ sinon $\mathrm{ETR}<\mathrm{ETP}=\mathrm{RU}+\mathrm{P}$. À chaque pas de 


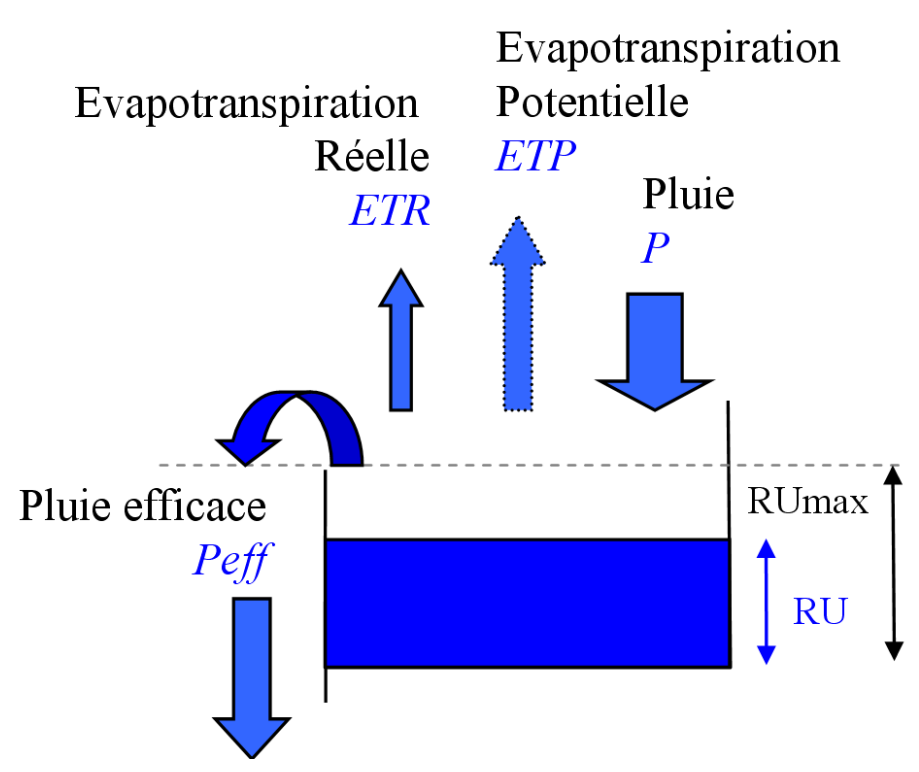

Figure 3. Modèle de Thornthwaite (1948).

Thornthwaite model (1948).

temps $n$, la pluie efficace est alors au maximum égale à P-ETR. L'algorithme qui en découle pour calculer l'ETR et la pluie efficace à chaque pas de temps est le suivant :

$$
\begin{gathered}
\text { ETR }^{\mathrm{n}}=\min \left(\mathrm{ETP}^{\mathrm{n}} ; \mathrm{P}^{\mathrm{n}}+\mathrm{RU}^{\mathrm{n}}\right) \\
\operatorname{Peff}^{\mathrm{n}}=\mathrm{P}^{\mathrm{n}}-\mathrm{ETR}^{\mathrm{n}}+\mathrm{RU}^{\mathrm{n}}-\mathrm{RU}_{\text {max }} \\
\text { Si Peff }{ }^{\mathrm{n}} \leq 0 \text { alors Peff } \\
\\
\mathrm{n} U^{\mathrm{n}+1}=\min \left(\mathrm{RU}_{\max } ; \mathrm{P}^{\mathrm{n}}+\mathrm{RU}^{\mathrm{n}}-\mathrm{ETR}^{\mathrm{n}}\right)
\end{gathered}
$$

Afin de réaliser ces calculs d'ETR et de pluie efficace pour chaque maille, il a été nécessaire de procéder à une spatialisation des données, notamment les données de pluviométrie et d'ETP. Ce dernier paramètre a été acquis auprès de Météo-France qui le calcule en un seul point de l'île (station du Lamentin) selon la formule de Penman-Monteith (ALLEN et al., 1998; MONTEITH, 1965; http://www.fao.org/docrep/x0490e/ x0490e06.htm).

\subsection{Pluviométrie et évapotranspiration potentielle-Discrétisation des cartes et création de matrices de coefficients}

De nombreux auteurs considèrent que la surface élémentaire représentative des phénomènes hydrologiques majeurs tels que le ruissellement, l'évapotranspiration et l'infiltration est d'environ un $\mathrm{km}^{2}$ (WOOD et al., 1988; WOOD et al., 1990). À l'intérieur de cette surface, l'hypothèse d'un continuum homogène peut être formulée pour la modélisation hydrologique. Dès lors, une grille avec un pas d'espace de
$1 \mathrm{~km}$ a été choisie pour discrétiser les diverses cartes. Ce choix, en ce qui a trait à l'échelle spatiale, semble bien justifié à la Martinique, pour deux raisons principales :

- d'une part, compte tenu des processus physiques (et notamment les précipitations) qui y sont observés, à l'échelle temporelle (pas de temps journalier) retenue dans le cadre du présent travail de recherche. En effet, en Martinique, les précipitations sont essentiellement orographiques. La composante convective est surtout significative en saison sèche. Si l'échelle spatiale de l'averse convective est alors plutôt celle du versant (20 à $30 \%$ de kilomètre carré environ), l'échelle temporelle associée est celle de la dizaine de minutes environ, ou de l'heure tout au plus. Au pas de temps journalier (plusieurs averses par jour), on accède à une échelle spatiale plus large. Le choix du kilomètre carré semble donc bien justifié en matière d'échelle minimale d'analyse des processus physiques étudiés au pas de temps journalier. Ce choix semble aussi nécessaire, compte tenu de la taille des bassins versants élémentaires en Martinique;

d'autre part, les données météorologiques sont relativement denses en Martinique (données de précipitations notamment; voir paragraphe suivant) et bien structurées spatialement (relief, vents dominants). Cela justifie a priori le choix de cette échelle spatiale relativement précise qui semble constituer un bon compromis entre des analyses trop fines ou trop grossières. Il est néanmoins délicat de démontrer de manière rigoureuse que la résolution « réelle » ou " physique " $\mathrm{du}$ modèle est celle $\mathrm{du} \mathrm{km}^{2}$, la résolution "physique " dépendant aussi de la variable considérée. Les résultats obtenus devront être considérés à l'échelle hydrologique, qui permet notamment d'agréger plusieurs mailles contigües du modèle, donc à une échelle sensiblement supérieure au $\mathrm{km}^{2}$.

L'opération de discrétisation a été réalisée avec le logiciel ArcMap@ (génération d'un fichier ASCII pour chaque carte) et les cartes ont ensuite été importées dans le logiciel Matlab(C) (traitement des données). Un exemple de cartes discrétisées (telles qu'elles sont utilisées en entrée du modèle numérique) est présenté sur la figure 2 .

La carte moyenne des isohyètes interannuelles (Figure 2) a été élaborée par Météo-France en 2006 (BRISSON, 2006, com. pers.) à partir des stations pluviométriques possédant au moins dix ans de données (entre 1915 et 2006) sur une période quelconque de 30 ans. Cette méthodologie se justifie tout d'abord par le fait que les analyses portant sur le changement climatique réalisées par Météo-France pour la Martinique n'ont pas mis en évidence de modifications de la pluviométrie sur la période 1915-2006. D’autre part, le calcul de moyennes sur une période de dix ans de données au minimum permet, dans le contexte climatique de la Martinique, d'atténuer l'effet d'une année particulièrement sèche ou, à l'inverse, humide. Cent trente stations ont ainsi été utilisées parmi les 220 qu’a pu 
compter la Martinique depuis le début des mesures en 1915, soit une densité moyenne d'une station pour $8 \mathrm{~km}^{2}$.

La carte de l'ETP moyenne interannuelle (Figure 2) a été élaborée par Météo-France (REGUIEG, 1986) à partir des chroniques de 26 postes climatologiques, pour lesquels des données étaient disponibles sur la période 1971-1985, et possédant au minimum dix ans de données. La mesure de l'évaporation était réalisée sur évaporomètre Piche pour les 26 postes et, en complément, à l'aide d'un bac A pour six d'entres eux. Le calcul de l'ETP était ensuite réalisé à l'aide de la formule de Bouchet (BOUCHET, 1964), les deux autres formules de calcul (Penman et Brochet-Gerbier) donnant un ordre de grandeur très similaire; en revanche, les formules de Thornthwaite et de Turc n'apparaissent pas adaptées à la Martinique. Cette carte met en évidence l'effet du relief sur l'ETP ainsi que les différences entre le nord et le sud de l'île. Étant donné la diminution de la température avec l'altitude et la diminution d'insolation liée à une plus forte nébulosité, l'évapotranspiration potentielle tend en effet à décroître avec l'altitude. La relation ETP-altitude ainsi obtenue $\left(-73 \mathrm{~mm} \bullet \mathrm{an}^{-1} \bullet 100 \mathrm{~m}^{-1}\right)$ est comparable à celle déterminée à l'île de la Réunion $\left(-67 \mathrm{~mm} \bullet \mathrm{an}^{-1} \bullet 100 \mathrm{~m}^{-1}\right.$ ) (ENILORAC, 1986). La diminution de l'ETP avec l'altitude est plus forte en saison humide qu'en période sèche et plus fraîche (carême).

Les précipitations présentent logiquement une variabilité spatiale plus forte que celle de l'évapotranspiration potentielle avec des écarts-types (normés par la moyenne arithmétique) respectivement de 0,36 et 0,25. L'écart-type absolu de la grille de la pluviométrie moyenne est nettement supérieur $(879 \mathrm{~mm})$ à celui de l'ETP $(375 \mathrm{~mm})$. Logiquement, les zones les plus arrosées, plus fraîches (altitude) et caractérisées par une nébulosité plus importante, coïncident avec les secteurs présentant la plus faible ETP tandis que les régions les moins arrosées, plus ensoleillées et/ou de moindre altitude présentent une plus forte ETP.

\subsection{Génération d'une chronique de pluie journalière pour chaque maille}

Quatorze zones pluviométriquement homogènes ont été délimitées par l'IRD sur la base des critères suivants : pluviométrie annuelle, nombre de jours de pluie et relief (MOBECHE, 2003). D'autre part, des chroniques de pluie journalière sont disponibles en une vingtaine de stations sur une période commune (1986-1997, période pendant laquelle un maximum de données hydrométriques est disponible; $c f . \$$ 4.2) (source : réseau pluviométrique de Météo-France et réseau pluviométrique ORSTOM/DIREN : http://www.martinique. environnement.gouv.fr/pluviometrie.html). Enfin, la carte des isohyètes moyennes interannuelles calculée sur la période 1915-2006 (BRISSON, 2006, com. pers.) a été utilisée.
Un pluviomètre de référence, choisi parmi ceux pour lesquels des chroniques sont disponibles pour la période retenue (1986-1997), a été attribué à chacune des 14 zones pluviométriquement homogènes $(\mathrm{ZPH})$. À chacune des mailles situées dans une même $\mathrm{ZPH}$ a été affectée la chronique du pluviomètre de référence de cette zone, corrigée par un coefficient multiplicatif destiné à tenir compte, entre autres, de l'altitude. Pour chaque maille, ce coefficient correcteur est égal au rapport entre la pluviométrie moyenne interannuelle établie à partir de la carte des isohyètes interannuelles, préalablement digitalisée au pas d'un kilomètre, et la pluviométrie moyenne interannuelle (1986-1997) mesurée au pluviomètre de référence.

\subsection{Génération d'une chronique d'ETP journalière pour chaque maille}

La carte des iso-ETP établie par Météo-France (REGUIEG, 1986) a été digitalisée sur le maillage kilométrique. La démarche mise en œuvre pour générer les chroniques journalières d'ETP est simplifiée par le fait d'une seule chronique d'ETP (station Météo-France du Lamentin; le calcul selon la formule de Penman-Monteith) est disponible. Pour chaque maille, l'ETP est obtenue au pas de temps journalier en multipliant la valeur journalière calculée à la station du Lamentin, par le rapport entre l'ETP moyenne annuelle établie à partir de la carte des iso-ETP et l'ETP moyenne annuelle à la station du Lamentin (1670 mm).

\section{5 Établissement de la carte des $R U_{\max }$}

La carte des $\mathrm{RU}_{\text {max }}$ (Figure 4a) a été établie en croisant la carte de la capacité de rétention des sols, hiérarchisée en " faible ", " moyenne ", " forte " (DESPRATS et al., 2003) et 25 mesures ponctuelles de réserve utile maximale réalisées par l'INRA (REGUIEG, 1986). Ainsi, à chaque classe a pu être affectée une valeur (respectivement $120 \mathrm{~mm}$ pour faible, $160 \mathrm{~mm}$ pour moyenne et $210 \mathrm{~mm}$ pour forte) (VITTECOQ et al., 2007).

\subsection{Génération de chroniques de Kc}

Pour les zones de cultures discrétisées à partir d'une carte simplifiée d'occupation des sols (Figure 4b, d'après CNASEA, 2004; DESPRATS et al., 2003), l'ETP a été corrigée pour tenir compte des besoins en eau spécifiques des plantes en fonction du stade phénologique (REGUIEG, 1986). Un coefficient cultural Kc a été introduit tel que : ETP corrigée $=\mathrm{Kc}^{*} \mathrm{ETP}$. Cette ETP corrigée est ensuite utilisée dans l'équation 1 et les équations suivantes. Des fonctions temporelles ont ainsi été construites pour représenter les variations saisonnières des 

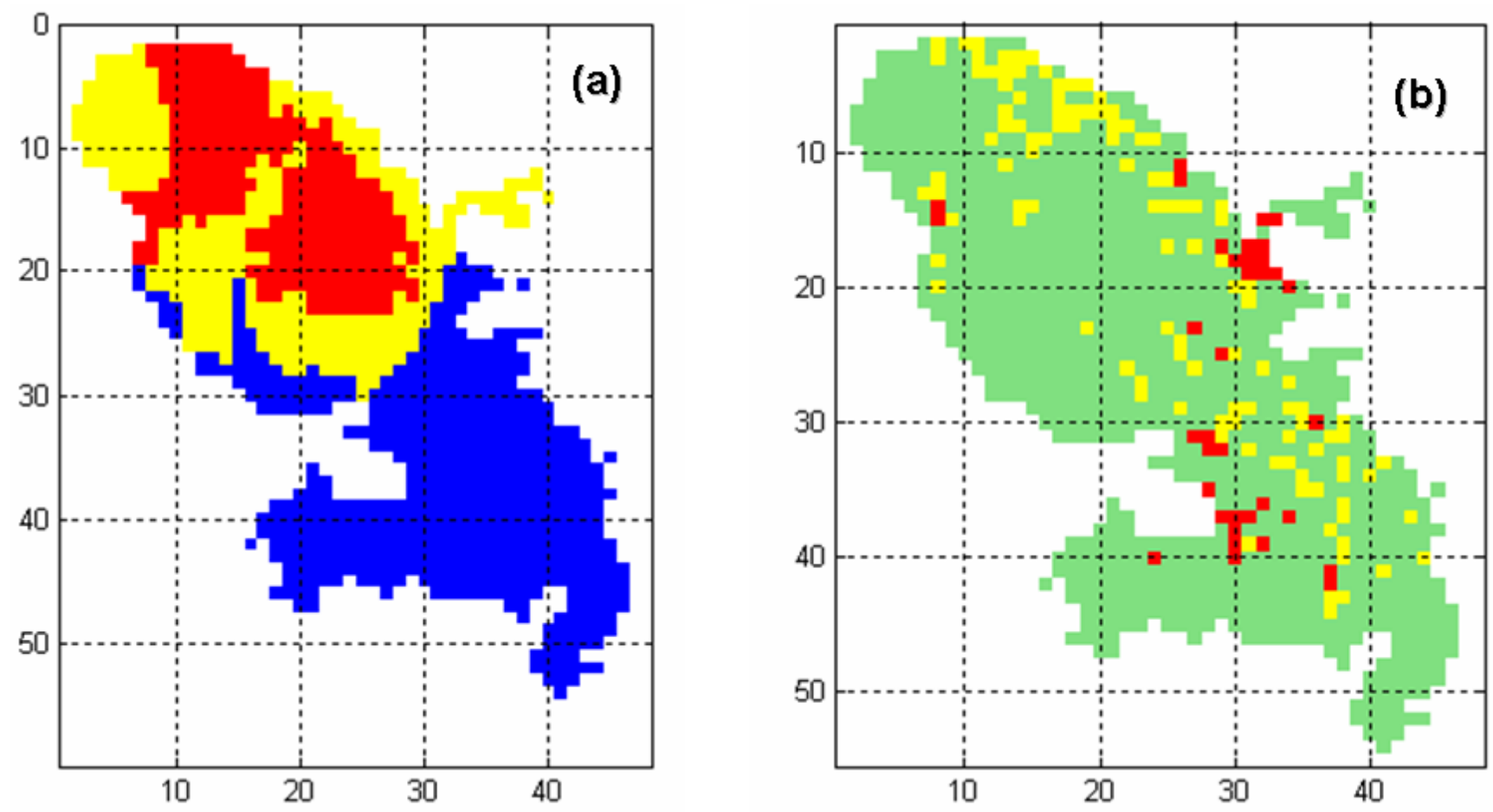

Figure 4. (a) : carte de la Réserve Utile maximale des sols; bleu = $120 \mathrm{~mm}$; jaune $=160 \mathrm{~mm}$; rouge $=210 \mathrm{~mm}$; (b) $:$ carte simplifiée de l'occupation des sols (jaune = banane; rouge = canne à sucre; vert = autre) (DESPRATS et al., 2003).

(a): map of maximum soil moisture reserve; blue $=120 \mathrm{~mm}$; yellow $=160 \mathrm{~mm}$; red $=210 \mathrm{~mm}$; (b): simplified landuse/landcover map (yellow = banana; red = sugar cane; green = other $)($ DESPRATS et al., 2003).

coefficients culturaux des deux principales cultures intensives existant en Martinique, la banane et la canne à sucre, à partir des valeurs disponibles pour chacun des stades phénologiques. Les autres types de couverts végétaux (forêts, pâturages, etc.), n'ont pas fait l'objet de l'affectation d'un coefficient cultural car celui-ci est très mal connu.

\subsection{Résultat: cartographie de la pluie efficace}

La pluie efficace a été calculée selon les modalités présentées ci-dessus à un pas de temps journalier, en chaque maille de $1 \mathrm{~km}^{2}$, sur la période 1986-1997. La figure 5 présente la carte des pluies efficaces moyennes interannuelles et la carte de l'évapotranspiration réelle moyenne interannuelle. Sur cette période 1986-1997, la valeur moyenne annuelle des pluies efficaces à l'échelle de l'île est de $1189 \mathrm{~mm}$ et celle de l'ETR est de $1272 \mathrm{~mm}$. Logiquement, la pluie efficace présente une plus forte variabilité spatiale (écart-type : $1063 \mathrm{~mm}$ ) que l'ETR (écart-type : $238 \mathrm{~mm}$ ). Les écarts-types normés montrent que le calcul de bilan hydrologique utilisé crée de la variabilité spatiale : la forte variabilité obtenue pour les pluies efficaces $(0,89)$ est nettement supérieure aux variabilités des termes d'entrée ( 0,36 pour la pluie et 0,25 pour l'ETP).

\section{VÉRIFICATION DU CALCUL DE LA PLUIE EFFICACE - DISCUSSION}

\subsection{Comparaison pluie efficace calculée - débits mesurés pour quelques bassins}

Des mesures hydrométriques sont disponibles sur plusieurs années (http://www.martinique.environnement. gouv.fr/hydrometrie.html) pour neuf bassins versants de la Martinique (Figure 1). Il est dès lors intéressant de comparer le cumul annuel de pluie efficace sur les mailles correspondantes du modèle et le cumul du débit mesuré à l'exutoire sur la même période temporelle (en tenant compte, le cas échéant, des prélèvements anthropiques, ou des rejets significatifs, s'il y en avait), afin de vérifier que ces deux variables sont du même ordre de grandeur, aux variations de stocks (sols, zone non saturée, aquiferres) et aux éventuels débits non jaugés (fuites en mer, écoulements souterrains) près (BREDEHOEFT, 2007).

En Martinique, ce type de comparaison est justifié car les stations hydrométriques contrôlent en général à la fois les écoulements de surface et souterrains, les aquifères étant connectés aux rivières et leurs niveaux d'eau contrôlés par la 

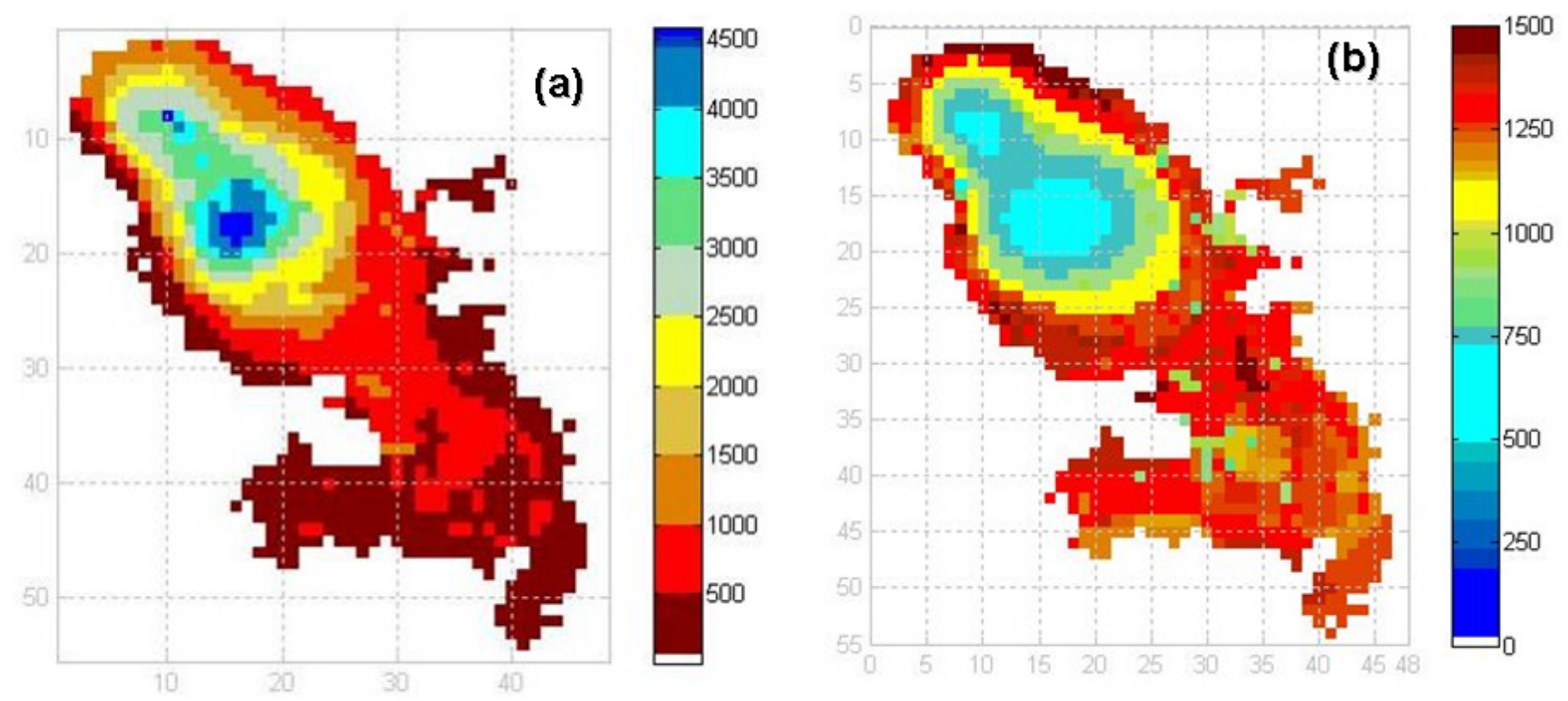

Figure 5. (a) : carte de la pluie efficace moyenne interannuelle, calculée sur la période 1986 - 1997 (en mm); (b) carte de l'évapotranspiration réelle moyenne interannuelle, calculée sur la période 1986 - 1997 (en mm).

(a): 1986 - 1997 computed mean annual efficient rainfall (mm); (b) 1986 - 1997 computed mean annual actual evapotranspiration (mm).

topographie. Les écoulements directs vers la mer, sans transit au sein des cours d'eau, sont restreints à la frange littorale et d'une importance a priori modeste dans la plupart des régions de l'île; seuls certains secteurs limités, une partie du flanc ouest de la montagne Pelée, par exemple, manifestent des écoulements directs des aquiferes en mer. En outre, les variations de stock d'une année hydrologique à l'autre, tant au sein des sols (AMEMA, 1982 à 1999 et MÉTÉO-FRANCE, 2000 à 2008) que des aquiferes (OLLAGNIER, 2008), sont peu importantes en regard des flux.

Les résultats obtenus sont présentés sur la figure 6 . Chaque point représente une année hydrologique complète (de juin à mai) comprise entre 1986 et 1997 (le nombre d'années disponibles varie selon les points de mesure). Des symboles différents permettent de distinguer chaque station hydrométrique. En tenant compte du fait que les mesures de débit ont une précision de $15 \%$ environ, on constate une relativement bonne corrélation entre les deux variables, pluies efficaces annuelles calculées et lame d'eau écoulée. Ce résultat conforte la méthodologie qui a été développée et les paramètres qui ont été utilisés pour calculer la pluie efficace; rappelons en effet que la méthodologie ne comporte aucun calage de paramètres et est basée sur un modèle hydrologique, avec une approche relativement sommaire, notamment en matière de bilan d'énergie.

On note néanmoins une tendance du modèle à légèrement surestimer (de 16 à $17 \%$ environ) les pluies efficaces pour la majorité des stations hydrométriques et des années. Ce biais apparent ne semble lié, en première analyse, ni à la taille des bassins versants, ni à leur localisation géographique ou leurs caractéristiques hydrométéorologiques. Ce dernier point concorde avec la connaissance hydrogéologique locale qui exclut des pertes significatives en mer (débits non contrôlés par les stations de jaugeage). Il n'est pas non plus lié à certaines années hydrologiques spécifiques.

En l'absence a priori de biais systématique conduisant à une surévaluation de la pluviométrie ou à une sous-estimation du débit des cours d'eau, c'est très vraisemblablement le calcul de l'évapotranspiration qui devrait être amélioré. La raison la plus probable tient au fait que ce modèle préliminaire ne prend pas en compte, en dehors des cultures intensives telles que la canne et la banane, d'autres types de couverts végétaux (forêts notamment) susceptibles d'évapotranspirer plus que la végétation de référence (herbe haute de $0,12 \mathrm{~m}$ ). Ainsi, les bassins versants de Petit-Bourg et Fontane, situés dans le sud de l'île et comportant relativement peu de forêt et des aires cultivées en banane et en canne relativement importantes, présentent une bonne adéquation pluie efficace - volume écoulé à l'exutoire. Les autres bassins versants comportent tous une part significative de zones non cultivées, et notamment des zones boisées qui requerraient en toute rigueur l'utilisation d'un coefficient de type Kc pouvant être supérieur à 1 pendant une partie de l'année et ainsi induire une évapotranspiration potentielle supérieure à celle calculée pour la végétation de référence. On note d'ailleurs que l'écart entre les pluies efficaces calculées et le débit mesuré à l'exutoire croît avec la pluie efficace tout comme l'écart entre les évapotranspirations 


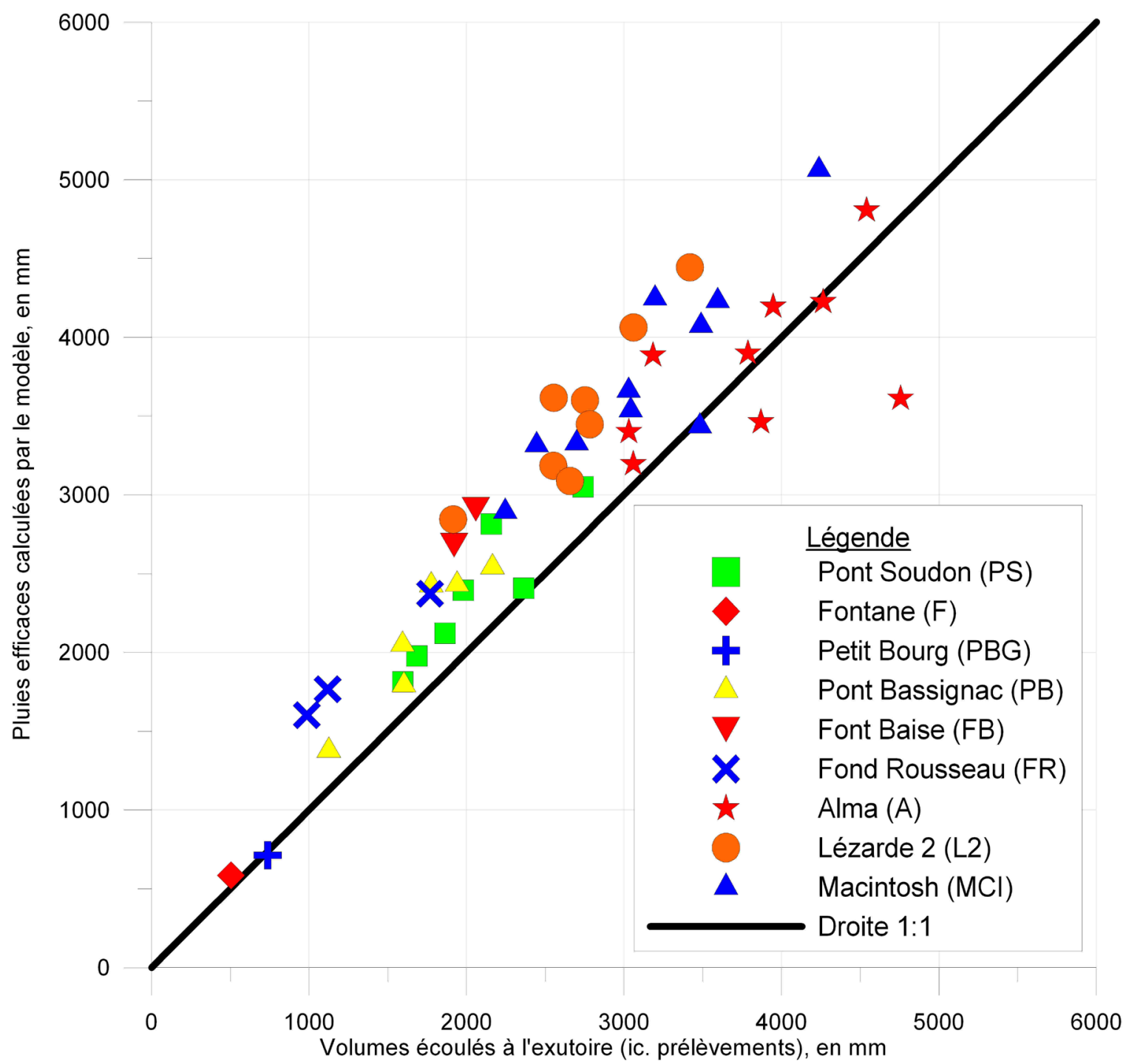

Figure 6. Comparaison Pluie efficace - débit à l'exutoire par année hydrologique (cumul juin - mai) pour neufs bassins versants instrumentés.

Comparison between computed efficient rainfall and observed annual discharge per hydrological year (June-May) for the nine ganged basins.

réelles des bassins forestiers et non forestiers croît avec la pluie selon la courbe mondiale de ZHANG et al. (2001). Cette interprétation préliminaire méritera néanmoins d'être approfondie car on ne dispose que d'une année pour ces deux bassins qui appartiennent en outre à une zone climatique assez différente des autres.

Par ailleurs, ce biais n'est a priori pas lié au fait que le modèle ne simule pas le stress hydrique $(\mathrm{ETR}=\mathrm{ETP}$ tant qu'il y a de l'eau dans le réservoir). En effet, la prise en compte du stress hydrique aurait tendance à diminuer l'ETR dans les conditions relativement sèches, donc pour les bassins versants les mieux simulés du sud de l'île, alors que ce sont les bassins versants les moins susceptibles d'être soumis au stress hydrique qui montrent un biais.

Cette nécessité de prendre notamment en compte les zones boisées pour calculer l'ETP suggère que les évaluations actuelles de l'ETP proposées en contexte tropical humide (par exemple : Martinique : REGUIEG, 1986; Réunion : ENILORAC, 1986), et ,par conséquent aussi, les évaluations de l'ETR sont sensiblement sous-estimées, au moins dans les secteurs comportant une proportion importante de couvert forestier.

La comparaison pluies efficaces - débits écoulés n’a été effectuée qu'au pas de temps annuel car c'est seulement à cette 
échelle de temps qu'elle est pertinente. En effet, à des échelles temporelles inférieures, la comparaison ne peut plus se faire en négligeant les effets liés au stockage dans les différents compartiments des hydrosystèmes (sols, zone non saturée, aquiferes). Aux stations de jaugeage disponibles dans le cadre du présent article, situées en général dans la partie aval des bassins versants, la prise en compte de ces effets nécessiterait une complète modélisation hydrologique (qui, néanmoins, conduirait à intégrer d'autres incertitudes). À titre d'illustration, la figure 7 présente les résultats obtenus au pas de temps mensuel à la station de jaugeage Macintosh (MCI). Sur la période décembre - avril, correspondant à la période de carême (saison sèche), le débit écoulé est quasi systématiquement supérieur aux pluies efficaces, ce qui est interprété comme un soutien par le drainage des aquifères au débit des rivières. Le reste de l'année (et particulièrement en juin-juillet qui correspond au début de la saison des pluies, mais aussi à la période pendant laquelle la lame d'eau écoulée reste encore modeste, donc la sensibilité de la comparaison pluies efficaces/écoulements est meilleure) les pluies efficaces sont égales ou supérieures aux débits écoulés, ce qui met ainsi en évidence le stockage dans les différents compartiments de l'hydrosystème, dont les aquifères.

\subsection{Evapotranspiration réelle et pluies efficaces}

Les cartes de la figure 5 et de la figure 8 présentent un aperçu des résultats des calculs issus de la méthodologie présentée ci-dessus et restituent, tant dans le détail que globalement, les caractéristiques hydroclimatiques de la Martinique, et notamment ses grands ensembles constitutifs :

- reliefs du centre et du nord, très arrosés, soumis à une moindre évapotranspiration potentielle que le reste de l'île et générant donc les pluies efficaces les plus élevées, avec des mailles dépassant les $4500 \mathrm{~mm}$ annuels. En moyenne interannuelle, l'ETR est quasi égale à l'ETP sur une part significative du nord de la Martinique, d'une part en raison des précipitations importantes et relativement bien réparties temporellement, dues principalement aux effets orographiques et, d'autre part, et dans une moindre mesure, en raison de la forte capacité de rétention hydrique des sols,

- restitution de l'effet de foehn (chinook) observé sur la côte sous le vent, notamment de Fort-de-France au canal de la Dominique et de la dissymétrie est-ouest des pluies efficaces,

- littoraux du sud de l'île et zones à faible relief (presqu'îles de la Caravelle et de Sainte-Anne) présentant de très faibles pluies efficaces (inférieures à $500 \mathrm{~mm} \bullet \mathrm{an}^{-1}$ ) et une évapotranspiration réelle nettement inférieure à l'évapotranspiration potentielle (zone à risque de stress hydrique).

\subsection{Conclusion-Perspectives}

L'approche proposée est relativement robuste et simple à mettre en œuvre, comparativement à des modèles plus détaillés mais de fait plus complexes car nécessitant plus de paramètres (BATELAAN et DE SMEDT, 2001). Elle ne requiert que peu de données, qui sont en outre facilement accessibles. Elle peut être utilisée dans de nombreuses autres régions du monde, notamment celles où des produits tels que ceux distribués par Météo-France ne sont pas disponibles ou sont élaborés à une échelle non compatible avec celle des besoins ou de la variabilité spatiale des processus qui s'y produisent; il s'agit en particulier des régions à relief marqué, présentant une forte variabilité spatiale de la pluviométrie et/ou de l'évapotranspiration.

Il est recommandé de mettre en œuvre une procédure de validation/critique du calcul des pluies efficaces telle que celle proposée ici afin de s'assurer de la qualité des résultats. La mise en regard des pluies efficaces interannuelles et des lames d'eau écoulées est tout particulièrement adaptée, en considérant bien entendu les particularités hydrologiques et hydrogéologiques des bassins versants jaugés utilisés pour ce faire. À cet égard, il apparaît important d'intégrer au modèle un coefficient cultural non seulement pour calculer l'évapotranspiration des surfaces cultivées, mais aussi pour évaluer celle des terrains en friche, et notamment celle des surfaces ayant une couverture forestière. Des améliorations en ce sens sont en cours d'implémentation dans le cadre de la modélisation réalisée en Martinique. La piste suivie est celle d'un calage général d'un seul coefficient Kc de type "forêt " pour l'ensemble de l'île, en considérant les surfaces boisées relatives à chaque bassin versant pour lequel des données hydrométriques sont disponibles.

Les résultats de cette modélisation des pluies efficaces en Martinique sont en cours de valorisation, notamment avec les différentes applications suivantes :

- la modélisation du fonctionnement des bassins versants, en déconvoluant la composante rapide et les composantes plus lentes associées aux circulations souterraines; les chroniques de pluies efficaces ainsi générées sont utilisées comme fonction d'entrée de ces modèles hydrologiques. Le résultat appliqué est principalement une évaluation des ressources en eau, et notamment des ressources en eaux souterraines exploitables à l'échelle des différentes régions de l'île;

- le traitement des chroniques piézométriques enregistrées dans le cadre du suivi patrimonial des ressources en eaux souterraines. 

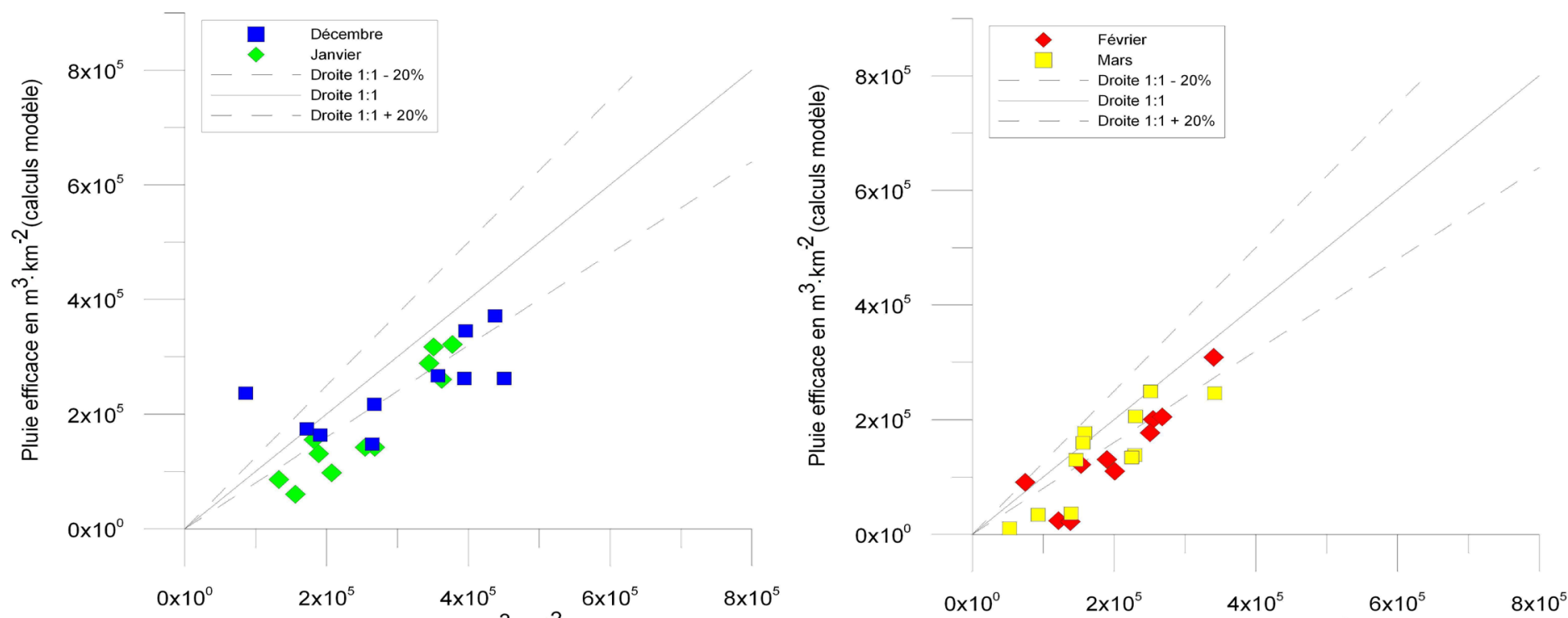

Volumes écoulés à l'exutoire, en $\mathrm{m}^{3} \cdot \mathrm{km}^{-2}$ (Mesures DIREN)
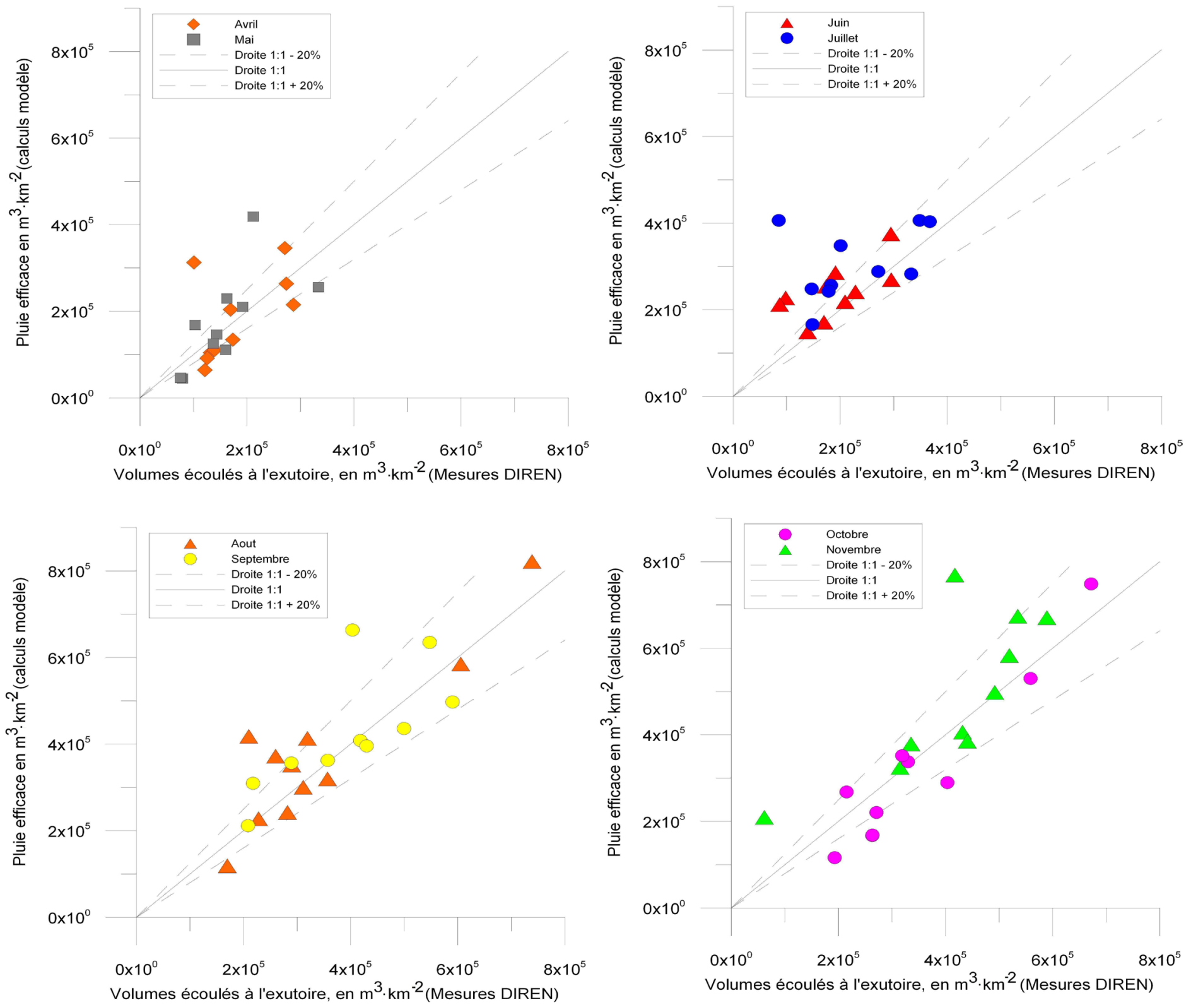

Figure 7. Comparaison des cumuls mensuels Pluie efficace - débit à l'exutoire à la station de jaugeage de Macintosh (MIC). Monthly comparison between computed efficient rainfall and observed annual discharge for the Macintosh (MIC) gauged basin. 


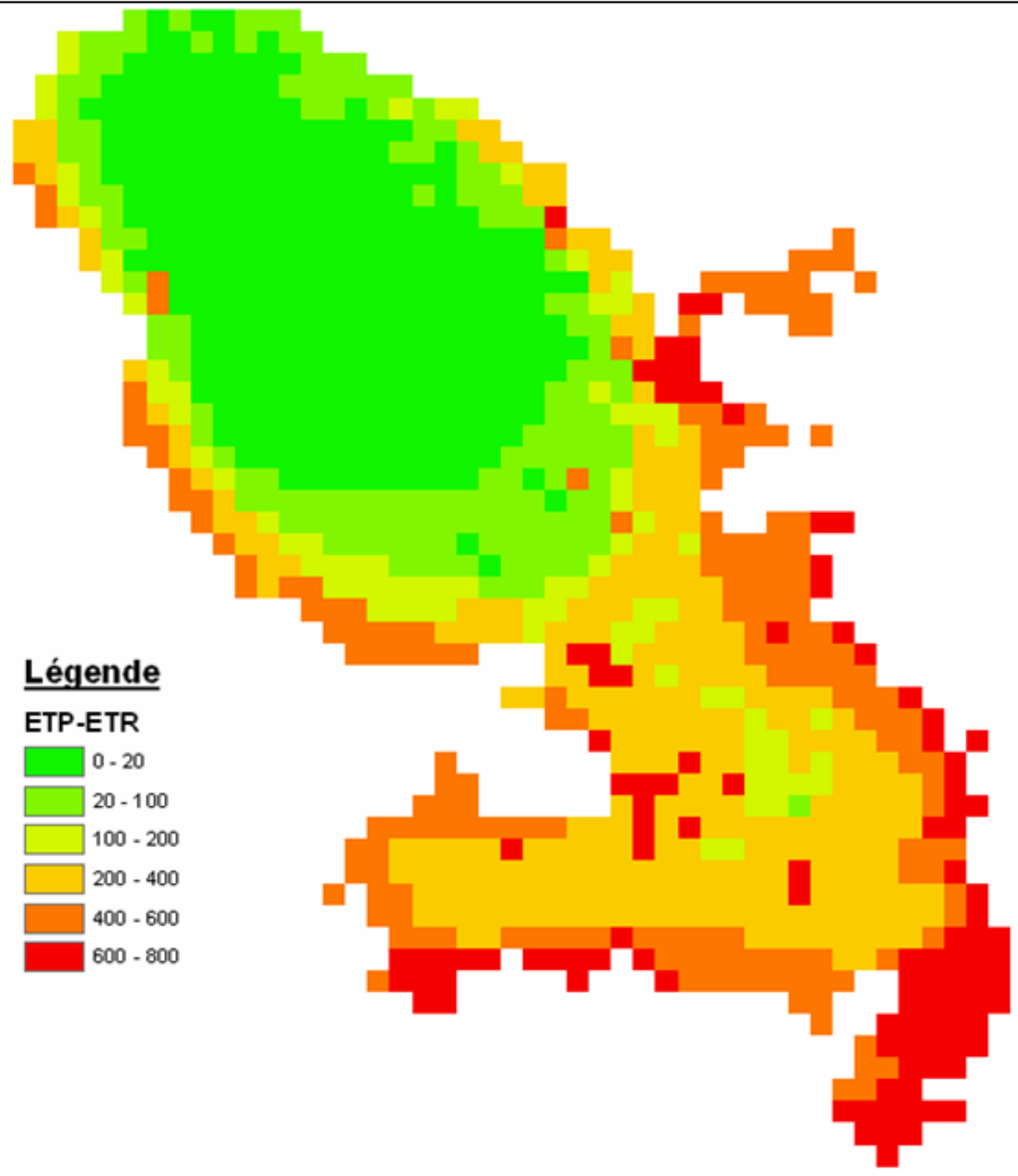

Figure 8. Différence ETP-ETR, en $\mathrm{mm}$. ETP minus ETR ( $\mathrm{mm})$.

\section{REMERCIEMENTS}

Le projet dans le cadre duquel cette méthodologie a été développée a été cofinancé par le Conseil Régional de la Martinique, la DIREN Martinique et par le BRGM, sur crédits de Service Public. Les auteurs souhaitent également remercier Y. Caballero (BRGM), pour sa contribution à la présentation des outils développés par Météo-France, P. Marras (DIREN) et A. Brisson (Météo-France), respectivement pour la mise à disposition des données hydrométriques et pluviométriques et de la carte des isohyètes moyennes annuelles.

\section{RÉFÉRENCES BIBLIOGRAPHIQUES}

ALLEN R.G., L.S. PEREIRA, D. RAES, M. SMITH (1998). Crop evapotranspiration - guidelines for computing crop water requirements. FAO Irrigation and Drainage paper,
56. Rome, Italy : Food and Agriculture Organization of the United Nations, ISBN 92-5-104219-5.

AMEMA (Association Météorologique de la Martinique) (1982 à 1999). Bulletin climatologique mensuel.

ALBERT P.H. et J. SPIESER (1999). Atlas climatique de la Martinique. Météo-France.

BATELAAN O. et F. DE SMEDT (2001). Wetspass: a flexible, GIS based, distributed recharge methodology for regional groundwater modelling. Dans : Impact of Human Activity on Groundwater Dynamics. GEHRELS et al. (Éditeurs.), vol. 269. IAHS, Wallingford, pp. 11-17.

BOUCHET R.J. (1964). Évapotranspiration réelle, évapotranspiration potentielle et production végétale. L'eau et la production végétale, I.N.R.A., 151-232.

BREDEHEHOEFT J. (2007). It is the discharge, Editorial. Groundwater, 45, 523, septembre-octobre 2007). 
CNASEA (2004). Atlas de la sole agricole. Département de Martinique, CNASEA Délégation Régionale de Martinique.

COLMET-DAAGE F., Z. BERNARD, J. GAUTHEYROU, M. GAUTHEYROU, F. LAGACHE, J. CRECY, A. POUMAROUX et A.PALLUD (1969). Carte des sols de la Martinique. BSA, ORSTOM, Pointe-à-Pitre.

COLMET-DAAGE F. (1969). Carte des sols des Antilles : Guadeloupe volcanique et Martinique au 1/20.000. ORSTOM, Antilles.

DESPRATS J.F., J.P. COMTE et G. PERIAN (2003). Cartographie par analyse multicritère des sols potentiellement pollués par organochlorés en Martinique - Phase 2. Rapport BRGM RP-52257-Fr.

DIREN. Données hydrométriques : http://www.martinique. environnement.gouv.fr/hydrometrie.html.

ENILORAC M.P. (1986). Contribution à l'étude de l'évapotranspiration potentielle sous climat tropical humide et d'altitude. Mémoire, Diplôme d'Agronomie Tropicale, Cirad, Montpellier, France, 119p.

GLOSSAIRE INTERNATIONAL D'HYDROLOGIE. http://www.cig.ensmp.fr/ -hubert/glu/HINDFRT.HTM.

GUISCAFRE J., J.C. KLEIN ET F. MONIOD (1976). Les ressources en eau de surface de la Martinique. Monographies hydrologiques - ORSTOM.

HABETS F., A. BOONE, J.L. CHAMPEAUX, P. ETCHEVERS, L. FRANCHISTEGUY, E.LEBLOIS, E. LEDOUX, P. LE MOIGNE, E. MARTIN, S. MOREL, J. NOILHAN, P. QUINTANA SEGUI, F. ROUSSETREGIMBEAU, P. VIENNOT (2008). The SAFRANISBA-MODCOU hydrometeorological model applied over France. J. Geophys. Res. D: Atmospheres, 113, D06113 (2008) 18.

IRD(2004).CartepédologiquedelaMartinique:http://collaboratif. ird.fr/ezpublish/index.php/IRDMartinique/content/ download/43425/138115/version/3/file/carte_pedo_ SIG972.pdf.

MÉTÉO-FRANCE (2000 à 2008). Bulletins climatologiques mensuels.

MOBECHE J.P. (2003). Homogénéisation et traitement des données pluviométriques de la Martinique par la méthode du vecteur régional. Cah. PRAM, octobre 2003, 15-19.
MONTEITH J.L. (1965). Evaporation and environment. Symp. Soc. Exp. Biol., 19, 205-224. Obtained from Forest Hydrology and Watershed Management - Hydrologie Forestiere et Amenagement des Bassins Hydrologiques (Actes du Colloque de Vancouver, août 1987) IAHSAISH,167,1987, 319-327.

NOILHAN J. et J.F. MAHFOUF (1996). The ISBA land surface parameterization scheme. Glob. Planet. Change, 13, 145-159.

NOILHANJ.etS. PLANTON (1989).Simpleparameterization of land surface processes for meteorological models. Month. Weather Rev., 117, 536-549.

OLLAGNIER S. (2008). Réseau piézométrique de Martinique, année 2007. BRGM/RP-56114- FR. 109 p., 7ill., 5ann.

ORSTOM/DIREN. Données pluviométriques avant 1992 (ORSTOM) et après 1992 (DIREN). http://www. martinique.environnement.gouv.fr/pluviometrie.html.

REGUIEG (1986). L'évapotranspiration et bilan hydrique en Martinique. Document Météo-France.

THORNTHWAITE C.W. (1948). An approach toward a rational classification of climate. Geogr. Rev., 38, 55-94.

VITTECOQ B., P. LACHASSAGNE, S. LANINI, B. LADOUCHE, J.C. MARECHAL et V. PETIT (2007). Élaboration d'un système d'information sur les eaux souterraines de la Martinique: identification et caractérisations quantitatives. Rapport final. BRGM/RP-55099-FR.

WESTERCAMP D., P. ANDREIEFF, P. BOUYSSE, S. COTTEZ, R. BATTISTINI (1989). Notice explicative, Carte géol. France (1/50 000), feuille Martinique - Orléans. Bureau de recherches géologiques et minières, $246 \mathrm{p}$.

WOOD E.F., M. SIVAPALAN, K. BEVEN et L. BAND (1988). Effects of spatial variability and scale with implications to hydrologic modeling. J. Hydrol., 102, 29-47.

WOOD E.F., M. SIVAPALAN et K. BEVEN (1990). Similarity and scale in catchment storm response. Rev. Geophys., 28, 1-18.

ZHANG.L, W.R., DAWES et G.R. WALKER (2001). Response of mean annual evapotranspiration to vegetation changes at catchment scale. Water Res. Rev., 37, 701-708. 